\title{
Tumor neuroendocrino en cérvix uterino: reporte de caso
}

\author{
Ither Sandoval-Díaz1, Ronald Hernández-Alarcón 1,2, Fernando Palacios-Cuervoa, \\ Andrea Calderón-Rivera ${ }^{a}$, Fátima Espinal-Reyes ${ }^{a}$, Esperanza Torres-Arones ${ }^{1}$, Andrea \\ Delgado-Elías. 3
}

${ }^{1}$ Servicio de Ginecología y Obstetricia, Hospital Nacional Edgardo Rebagliati Martins. ${ }^{2}$ Facultad de Ciencias de la Salud, Universidad Peruana de Ciencias Aplicadas. ${ }^{3}$ Médico Gineco-Obstetra. Lima, Perú.

a Alumno/a, Escuela de Medicina, Universidad Peruana de Ciencias Aplicadas, Lima, Perú.

\section{RESUMEN}

Los tumores neuroendocrinos de cuello uterino son extremadamente raros. Las mujeres con diagnóstico de carcinoma neuroendocrino de células pequeñas del cuello uterino tienen mayor frecuencia de metástasis en los ganglios linfáticos, invasión linfovascular, recurrencia y peor pronóstico en comparación con aquellos con otros tipos de neoplasias cervicales. Se presenta el caso de una mujer de 58 años, con un tiempo de enfermedad de seis años antes del ingreso, caracterizado por sangrado vaginal irregular posmenopáusica, además de sintomatología relacionada a anemia crónica. En el examen ginecológico, se evidenció tumoración de $4 \mathrm{~cm}$ que ocupaba tercio superior de vagina y protruía por el cérvix. Fue diagnosticado como mioma abortivo y enviada a estudio anatomopatológico. El resultado fue carcinoma neuroendocrino de células pequeñas grado III en el $90 \%$ y carcinoma epidermoide en el $10 \%$. La paciente fue sometida a histerectomía radical más salpingo-ooferectomía bilateral y linfadenectomía pélvica bilateral y para-aortica. El estudio anatomopatológico de la pieza quirúrgica encontró endometrio y miometrio comprometido por neoplasia maligna. Parametrios, anexos y ganglios linfáticos se encontraron libres de neoplasia. A la microscopía el resultado fue carcinoma neuroendocrino grado III (carcinoma de células pequeñas, infiltrante), con extensa embolia linfovascular. El estudio de inmunohistoquímica arrojó sinaptofisina positivo en las áreas con diferenciación neuroendocrina.

\section{PALABRAS CLAVE: Cáncer cérvico-uterino, carcinoma neuroendocrino, carcinoma de células pequeñas}

\section{SUMMARY}

Neuroendocrine tumors of the cervix are extremely rare. Women diagnosed with small cell neuroendocrine carcinoma of the cervix have a higher frequency of metastases in the lymph nodes, lymphovascular invasion, recurrence and worse prognosis compared to those with other types of cervical neoplasia. We report the case of a 58-year-old female, with a history of six years of postmenopausal irregular vaginal bleeding, in addition to symptoms related to chronic anemia. Gynecological examination showed a tumor of $4 \mathrm{~cm}$ that occupied the upper third of the vagina and protruded through the cervix initially diagnosed as an abortifacient myoma, and sent to histopathology study. $90 \%$ of the tumor was small cell neuroendocrine carcinoma grade III, and the remaining $10 \%$ was squamous cell carcinoma. The patient underwent into a radical hysterectomy plus bilateral salpingo-oophorectomy, and bilateral pelvic and para-aortic lymphadenectomy. Histopathologic examination of the surgical specimen found endometrium and myometrium compromised by malignancy. Parametrium, annexes and lymph nodes were free of neoplasia. At microscopy, the result 
was a grade III neuroendocrine carcinoma (small cell carcinoma, infiltrating), with extensive lymphovascular emboli. The immune-histochemical study showed synaptophysin positive in areas with neuroendocrine differentiation.

\section{KEY WORDS: Cervico uterine cancer, neuroendocrine carcinoma, small cell carcinoma}

\section{INTRODUCCIÓN}

El cáncer de cérvix es la quinta neoplasia más frecuente en el mundo y ocupa el segundo lugar en incidencia en mujeres, después del cáncer de mama (1). La Organización Mundial de la Salud (OMS) ha estimado que aparecen 500.000 casos nuevos por año, de los cuales el $80 \%$ ocurren en países en desarrollo (2). En el Perú, el cáncer de cérvix es la neoplasia de mayor prevalencia en mujeres (3). Los tipos histológicos más comunes del cáncer de cérvix son el carcinoma escamoso y el adenocarcinoma, teniendo como principal etiología la infección con el Virus Papiloma Humano (HPV). Otros tipos histológicos incluyen el carcinoma adenoide quístico, tumor neuroendocrino y el carcinoma indiferenciado.

Los tumores neuroendocrinos son comúnmente encontrados en el tracto gastrointestinal y en los pulmones, y son poco frecuentes en el cuello uterino. La clasificación del carcinoma neuroendocrino del cuello del útero incluye cuatro subtipos histológicos: carcinoma de células pequeñas, carcinoma de células grandes, tumor carcinoide clásico, y tumor carcinoide atípico (4). Cada una de las cuatro categorías histológicas puede estar asociada a un cáncer in situ, carcinoma invasivo de células escamosas o adenocarcinoma de cuello uterino (5).

El carcinoma neuroendocrino de células pequeñas del cuello uterino tiene mayor frecuencia de metástasis en los ganglios linfáticos, invasión linfovascular, recurrencia y peor pronóstico en comparación con otros tipos de neoplasias cervicales (6). En las últimas décadas, se ha reportado un aumento de la incidencia de estos tumores, lo que puede ser reflejo de una mayor disponibilidad de herramientas diagnósticas. Pese a ello, los tumores neuroendocrinos del cuello uterino siguen siendo considerados raros, incluyendo limitados datos prospectivos para guiar las decisiones terapéuticas, e información limitada sobre la sobrevida a largo plazo (5), sobre todo en países en desarrollo.

El objetivo de esta comunicación es dar a conocer el diagnóstico y manejo de una paciente con carcinoma neuroendocrino del cuello uterino.

\section{Caso clínico}

Mujer de 58 años, nulípara, sin antecedentes de importancia, quien presenta un tiempo de enfermedad de 6 años de inicio insidioso y curso progresivo, con episodios agudos de ginecorragia. El cuadro se inicia 6 años antes de su ingreso presentando sangrado vaginal 2 años después de la menopausia de la paciente y cursó de manera intermitente con una duración de 3 a 4 días cada 4 meses. Tres semanas antes de su ingreso la paciente refiere ginecorragia persistente y profusa, mareos, sensación de desvanecimiento y palidez, por lo cual, acude a emergencia donde se decide su hospitalización.

En el examen físico se encontró una paciente en regular estado general, con obesidad mórbida y palidez de piel y mucosas. El resto del examen no mostró alteraciones significativas. En el examen ginecológico se encontró genitales externos de acuerdo a edad con huellas de sangrado reciente. A la especuloscopía se evidenció tumoración de 4 $\mathrm{cm}$ de diámetro aproximadamente, superficie regular, sangrante al roce, color rojo vinoso que ocupa canal vaginal en tercio superior no dejando observar el cuello uterino. Al tacto vaginal se palpó tumoración algo friable con pedículo de aproximadamente $0,5 \mathrm{~cm}$ que ingresa a canal endocervical, no pudiéndose delimitar útero y anexos por obesidad y disconfort de la paciente. Se concluyó el diagnóstico de mioma abortivo y anemia crónica. La tumoración fue extraída por torsión sin intercurrencias y enviada a anatomía patológica (Figura 1).

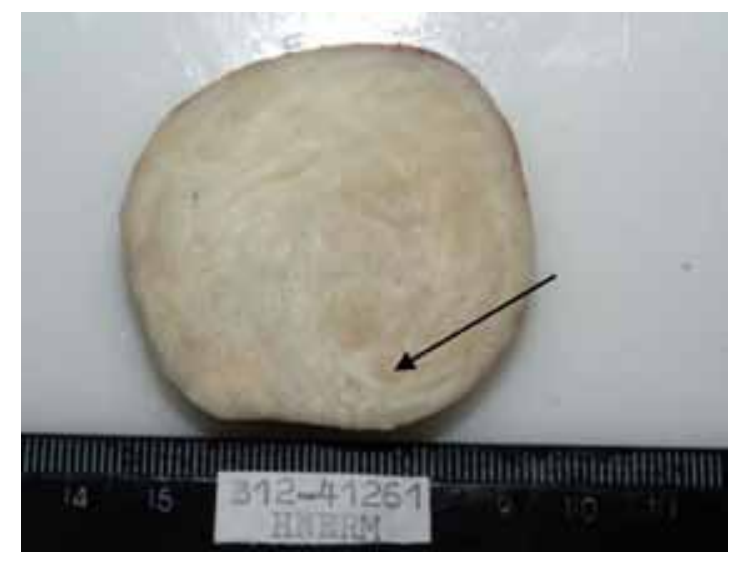

Figura 1. Foto macroscópica, se aprecia nódulo miomatoso de superficie arremolinada, la flecha señala área irregular correspondiente a componente carcinomatoso. 
La ecografía transvaginal mostró un útero retroverso de $78 \times 52 \times 44 \mathrm{~mm}$, miometrio heterogéneo con múltiples imágenes nodulares hipoeicoicas en cara anterior y posterior, línea endometrial mal definida por interposición de las mismas, fondo de saco de Douglas libre, ovarios involutivos sin alteraciones ecográficas. En la resonancia magnética nuclear (RMN) se encontró útero con miomas pequeños (mayor de $3 \mathrm{~cm}$ de diámetro entre cuerpo y cuello) y con algunos focos adenomiósicos.

Los exámenes de laboratorio mostraron una glucosa de $110 \mathrm{mg} / \mathrm{dL}$, urea $17,3 \mathrm{mg} / \mathrm{dL}$, creatinina $0,41 \mathrm{mg} / \mathrm{dL}$, anticuerpos para $\mathrm{VIH}$ no reactivo, $y$ hemoglobina de $5,7 \mathrm{mg} / \mathrm{dl}$ con microcitosis e hipocromía en el frotis de sangre periférica.

El informe de anatomía patológica de la tumoración calificada como mioma abortivo fue carcinoma neuroendocrino de células pequeñas grado III en el $90 \%$ y carcinoma epidermoide en el $10 \%$. La paciente fue sometida a histerectomía radical, salpingo-ooferectomía bilateral, linfadenectomía pélvica bilateral y para-aórtica. En los hallazgos intraoperatorios no se encontró líquido libre en cavidad, útero de $10 \mathrm{~cm}$ en anteversoflexión con superficie deformada por presencia de miomas intramurales que alteran la arquitectura uterina, la mayor de 2 $\mathrm{cm}$ en cara anterior. Ambos anexos sin alteraciones macroscópicas. Fondo de saco anterior y posterior libre. No se palpan adenopatías pélvicas ni para aórticas, los parametrios normales.

El estudio anatomopatológico del útero mostró a la macroscopia endometrio y miometrio comprometido por neoplasia maligna. La microscopía concluyó un carcinoma neuroendocrino grado III (carcinoma de células pequeñas, infiltrante) nacido de la parte alta del canal endocervical de $1,5 \mathrm{~cm}$ de extensión, $1,2 \mathrm{~cm}$ de profundidad, con extensa embolia linfovascular (Figura 2). En el estudio de inmunohistoquímica arrojó sinaptofisina positivo en áreas con diferenciación neuroendocrina y cromogranina negativo (Figura 3 ).

La paciente evolucionó favorablemente siendo dada de alta para inicio de radioterapia, acudiendo a una sesión luego de lo cual no vuelve a retornar a la institución para seguimiento y continuidad de tratamiento.

\section{DISCUSIÓN}

La presentación del caso reportado expuso una sintomatología de sangrado vaginal anormal y una tumoración simulando un diagnóstico de mioma abortivo. La clínica de estos tumores suele presentarse con síntomas abdomino-pélvicos como sangrado o flujo vaginal, dolor pélvico opresivo en la pelvis como fue el caso de la paciente reportada. Es raro que las pacientes sean asintomáticas o que resulte de un hallazgo ginecológico casual tras la prueba de Papanicolaou (7). Cabe resaltar que este tumor tiene alta incidencia de metástasis temprana por lo que el primer síntoma podría ser un síndrome paraneoplásico o consuntivo. En el caso reportado, a pesar del mal pronóstico por el tipo celular, no se encontró metástasis, y el principal motivo de consulta fue el sangrado vaginal y la sintomatología asociada a la anemia.

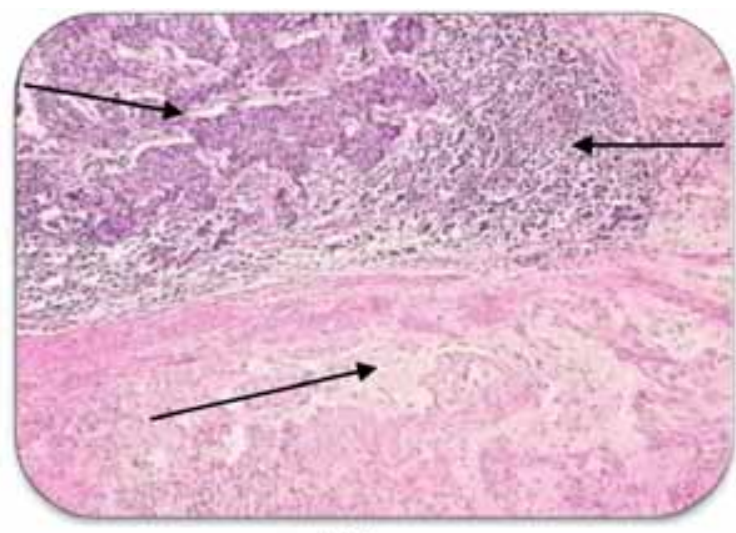

(a)

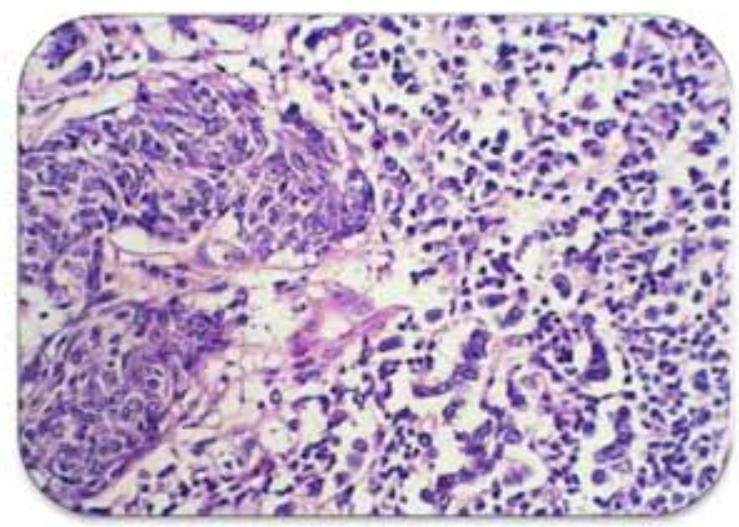

(b)

Figura 2: (a) Leiomioma (parte inferior), carcinoma epidermoide (arriba a la derecha) y carcinoma neuroendocrino (arriba a la izquierda). (b) Carcinoma epidermoide (derecha) y carcinoma neuroendocrino (izquierda). 


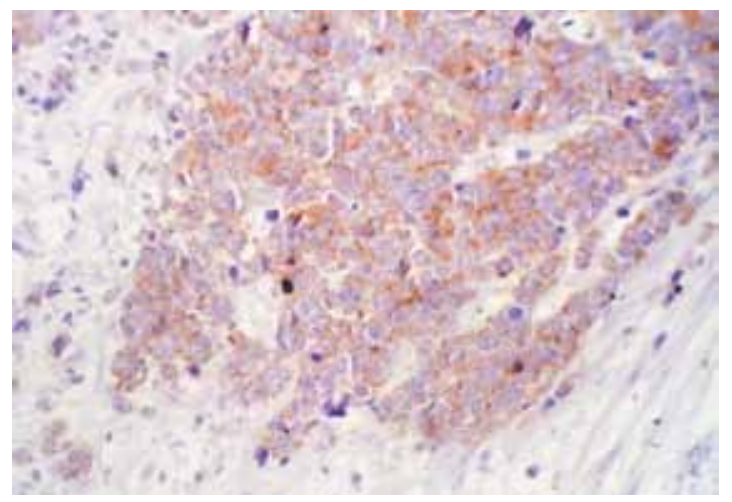

Figura 3. Sinaptofisina positiva en componente neuroendocrino.

Para el diagnóstico de estos tumores es importante realizar una buena anamnesis y examen clínico con la finalidad de enfocar adecuadamente los exámenes auxiliares que comprobarían la sospecha planteada. Dentro de los exámenes complementarios se destaca la biopsia, la cual especifica el diagnóstico definitivo como en el caso de la paciente, tanto del mioma extraído en emergencia como de las piezas operatorias durante la intervención.

En la microscopía con la tinción hematoxilina eosina, en estos tumores las células se posicionan en forma de racimos o trabéculas separadas por un estroma fibrovascular. En el caso de la paciente el diagnóstico histológico fue el de un carcinoma neuroendocrino puro de células pequeñas grado III. El carcinoma de células pequeñas del cuello uterino es el subtipo más común y agresivo de los tumores neuroendocrinos del cérvix, representando al $2 \%$ de todos los carcinomas de cuello uterino (5). Su alto grado de agresividad y la temprana propagación metastásica nos indica el pobre pronóstico del carcinoma de células pequeñas al momento del diagnóstico. Las metástasis de mayor frecuencia son las de hueso, cerebro, hígado y médula ósea. Por este motivo, además del estadiaje tradicional del cáncer de cuello del útero, en las pacientes se debe realizar un estudio radiológico óseo, hepático y cerebral, así como un aspirado y una biopsia de medula ósea en busca de posible metástasis (4).

Reportes previos han mostrado que las pacientes diagnosticadas con carcinoma de células pequeñas de cérvix tienen mayor frecuencia de metástasis a nódulos linfáticos, invasión linfo-vascular, recurrencia, y pobre pronóstico comparado con aquellos otros tipos de cáncer malignos de cérvix (8). Siendo una de las neoplasias ginecológicas más agresivas La tasa de supervivencia a 5 años para las pacientes con este tumor ha sido reportada de $14 \%$ en estadios avanzados, por lo tanto, el carcinoma cervical neuroendocrino de células pequeñas supone un desafío terapéutico para el oncólogo.

El criterio de estadificación de la FIGO (International Federation of Gynecology and Obstetrics) es importante en este tipo de cáncer de cuello uterino. En estadios tempranos de la enfermedad, se encontró que pacientes con tumores pequeños $(<2$ $\mathrm{cm}$ ) tuvieron significativamente mejores tasas de supervivencia que aquellas con tumores más gran$\operatorname{des}(\geq 2 \mathrm{~cm})(6)$.

En el caso reportado no se halló inicialmente signos de metástasis, y los ganglios eran negativos para cáncer. Por otro lado, para identificar el origen celular de la tumoración, se realizó estudios de inmunohistoquímica, en donde aproximadamente el $88-100 \%$ de estos tumores dará positivo para marcadores, como: enolasa neuro-específica (sensibilidad de 75\%), sinaptofisina (sensibilidad 60-90\%), cromogranina (sensibilidad $50-76 \%$ ) y CD56 (molécula de adhesión celular neural) considerado el marcador más sensible para el diagnóstico de células pequeñas (sensibilidad $71-92 \%)(9,10,11)$. La sinaptofisina tuvo positividad en áreas con diferenciación neuroendocrina en la biopsia de la paciente, mientras que la enolasa neuro-especifica tuvo resultados dentro de los valores normales $(13,23$ $\mathrm{ng} / \mathrm{dL}$ ). Se presume que la incidencia de los tumores neuroendocrinos es mayor a la reportada y por ende, se aconseja el estudio inmuno-histoquímico de toda neoplasia de cuello uterino con el fin de clasificarlas e identificarlas en estadios tempranos dada la precocidad de generación de metástasis a distancia.

Existen factores pronósticos clínicos patológicos reconocidos a tener peor pronóstico en el desenlace de la enfermedad, como tabaquismo, patrón polipoide, presencia de ganglios positivos, tratamiento con cirugía, y estadio FIGO avanzado $(6,12)$. En el caso reportado no se encontró ninguno de estos factores. Sin embargo, los factores pronósticos inmunohistoquímicos también fueron evaluados. En estudios retrospectivos se determinó que los pacientes cuyos tumores presentaban cromogranina positivo tenían una tasa de supervivencia significativamente peor que aquellos que tenían la cromogranina negativo (13). Además que en otros estudios sugieren que los pacientes cuyos tumores que expresen HER-2/NEU negativo en combinación con EGFR (epidermal growth factor receptor) positivo igualmente presentaban un bajo pronóstico de supervivencia (12).

Actualmente no existen guías definidas para manejo de enfermedad avanzada de tumor neuroendocrino en cérvix uterino y se conoce que el tratamiento es dependiente del estadío de la enfermedad. Para estadíos limitados de enfermedad resecable se indica terapia combinada (cirugía seguida por quimioterapia o quimioradioterapia combinada), y para 
enfermedad avanzada no resecable metastásica (quimioterapia definitiva), y no metastásica (quimioradioterapia combinada) (14). La cirugía quedará indicada como terapia adyuvante para lesiones mayores a $4 \mathrm{~cm} \mathrm{(5).} \mathrm{Por} \mathrm{otro} \mathrm{lado,} \mathrm{en} \mathrm{un} \mathrm{metanálisis} \mathrm{se}$ demostró que la histerectomía radical es un factor independiente de supervivencia (15).

Dado el mal pronóstico de estas neoplasias, es importante identificar los factores asociados a la supervivencia en un esfuerzo por mejorar las estrategias de tratamiento (6). Debido a que el carcinoma neuroendocrino del cuello uterino se produce con poca frecuencia, es difícil realizar un ensayo clínico aleatorizado y controlado para determinar el tratamiento óptimo (6).

\section{CONCLUSIÓN}

El tumor neuroendocrino, el cual se debe tener en cuenta como diagnóstico diferencial de toda neoplasia de cérvix, requiere de estudios que clarifiquen la historia natural, los factores pronósticos y las intervenciones terapéuticas más apropiadas para este tipo de tumor.

Agradecimientos: Al Dr. Alonso Soto por su disposición y colaboración en el desarrollo del manuscrito. Al Dr. Alan Latorre Zúñiga por facilitarnos el acceso a las fotografías de la biopsia de la paciente.

\section{REFERENCIAS}

1. Es Salud (Seguro Social del Perú). Guía de práctica clínica de cáncer de cuello uterino: Introducción. Febrero 2011. pp 14-15. Disponible en: http://www.ginecologiaperu.com/files/guia_c_cuello_uterino2011_ essalud.pdf

2. Organización Mundial de la Salud (OMS). Salud de la mujer: Cáncer del cuello uterino. Nota descriptiva №334, Noviembre de 2009. Disponible en: http:// www.who.int/mediacentre/factsheets/fs334/es/

3. Análisis de la Situación del Cáncer en el Perú, 2013Lima. Ministerio de Salud, Dirección General de epidemiología 2013. pp 36-42. Disponible en: http:// www.dge.gob.pe/portal/docs/asis_cancer.pdf

4. Berek J. Ginecología de Novak: Cáncer de Cuello de Útero y Vagina. Wolters Kluwer Health España: 2008. pp 1459-510.
5. Gardner G, Reidy-Lagunes D, Gehrig P. Neuroendocrine tumors of the gynecologic tract: A Society of Gynecologic Oncology (SGO) clinical document. Gynecol Oncol 2011;122:190-8.

6. Chan J, Loizzi V, Burger R, Rutgers J, Monk B. Prognostic factors in neuroendocrine small cell cervical carcinoma: a multivariate analysis. Cancer 2003; 97:568-74.

7. de León Luis JA, Pintado MP, Hernández A, Carrasco MC, Chiva L, Lizarraga S, et al. Carcinoma neuroendocrino de cérvix uterino en estadio temprano. Prog Obstet Ginecol 2003; 46:171-6.

8. Demonte $M$, Prozzillo L, Martinez D, Soderini A, Norma G, Alcoba E, et al. Tumores neuroendocrinos de cérvix uterino: variedad células pequeñas. Controversias en Obstetricia y Ginecología 2012;19-27. Disponible en: https://intervalolibre.files.wordpress. com/2012/06/tumores_neuroendocrinos.pdf

9. Tian W, Zhang M, Shui R. Prognostic factors and treatment comparison in early-stage small cell carcinoma of the uterine cervix. Oncol Letters 2012;3:12530.

10. Cohen J, Kapp D, Shin J, Urban R, Sherman A, Chen $\mathrm{L}$, et al. Small cell carcinoma of the cervix: treatment and survival outcomes of 188 patients. Am J Obstet Gynecol 2010;203:347.e1-6.

11. Viswanathan $A$, Deavers $M$, Jhingran $A$, Ramirez $P$, Levenback $C$, Eifel $P$. Small cell neuroendocrine carcinoma of the cervix: outcome and patterns of recurrence. Gynecol Oncol 2004;93:27-33.

12. Straughn J, Richter H, Conner M, Meleth S, Barnes M. Predictors of outcome in small cell carcinoma of the cervix--a case series. Gynecol Oncol 2001;83:216-20.

13. Bifulco G, Mandato V, Giampaolino P, Piccoli R, Insabato L, de Rosa N, et al. Small cell neuroendocrine cervical carcinoma with 1-year follow-up: case report and review. Anticancer Res 2009;29:477-84.

14. McCluggage W, Kennedy K, Busam K. An immunohistochemical study of cervical neuroendocrine carcinomas: Neoplasms that are commonly TTF1 positive and which may express CK20 and P63. Am J Surg Pathol 2010;34:525-32.

15. Boruta DM 2nd, Schorge J, Duska L, Crum C, CastriIlon D, Sheets E. Multimodality therapy in early-stage neuroendocrine carcinoma of the uterine cervix. Gynecol Oncol 2001;81:82-7. 